\title{
On the accuracy of the Adaptive Cross Approximation algorithm
}

\author{
Alex Heldring, Eduard Ubeda, and Juan M. Rius
}

\begin{abstract}
- this contribution identifies an often ignored source of uncertainty in the accuracy of the Adaptive Cross Approximation (ACA) algorithm.
\end{abstract}

Index Terms-ACA, Fast methods, MoM, Integral Equations.

\section{INTRODUCTION}

$T^{1}$ HE Adaptive Cross Approximation (ACA) [1] is an algorithm that computes a low rank approximation to matrices or matrix sub-blocks. Due to its efficiency and its black-box nature (no prior knowledge of the matrix content or origin is necessary), it is gaining ground as a tool in accelerated electromagnetic simulations. As with all approximations, the ACA introduces an error that can only be known up to an order of magnitude, to be chosen by the user. With the growing popularity of the ACA, so grows the importance of assessing the reliability of the algorithm under all circumstances.

In this contribution, we intend to draw attention to one aspect of the ACA algorithm that introduces a variation in the actual error around the user-chosen error threshold of about one order of magnitude. This variation is triggered by an initial choice inside the algorithm which is not left to the user but instead hard-wired in the algorithm formulation. There is presently no known recipe for optimizing this choice and as we shall argue it is probably not possible to find one. This means that the ACA algorithm is, to some extent, a randomized algorithm, with a probability distribution for the true residual error.

The reason that these observations have not been made before, even though the ACA algorithm has been receiving ample attention for some time now, is twofold: Firstly, since the initial setting is hard-wired, the user will always obtain identical results on identical problems and the algorithm will seem perfectly deterministic to her. Secondly, the ACA is typically used to compress off-diagonal blocks of a larger linear system. How exactly the relative error in the blocks propagates into the solution of the linear system cannot be known but it will often be considerably smaller, as the matrix

Manuscript received February 25, 2015. This work was supported by the Spanish Ministry of Economy and Competitiveness under project TEC201347360-C3-1-P

All three authors are with Universitat Politècnica de Catalunya, c/Jordi Girona 1, 08034 Barcelona, Spain (phone: 934017216; fax: 934017232; email: heldring@tsc.upc.edu). is often dominated by the non-approximated on-diagonal blocks. So it is unlikely, though not impossible, that an error that is one order of magnitude larger than the user intended, will result in an error of the same magnitude in the final solution.

Remains to be mentioned that the possible unreliability of the estimated error in the ACA algorithm has been noted before, although without linking it to the initial choice and consequently without addressing its statistical nature, notably in [2] and [3].

\section{NUMERICAL EXAMPLE}

As an illustration, the ACA algorithm is invoked to compress the mutual interaction matrix of the two square PEC plates shown in Fig. 1, in the EFIE formulation and using RWG basis functions. The plates are discretized into 1160 basis functions each, and the working frequency is such that the plate edges span two wave lengths.

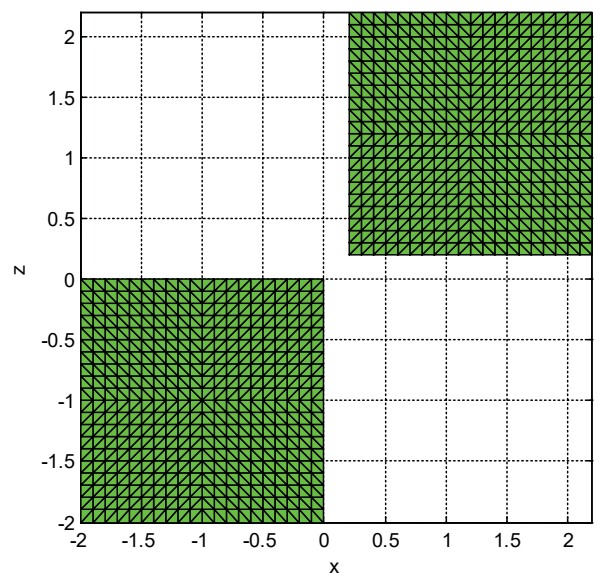

Fig. 1. Two square plates in the $\mathrm{x}-\mathrm{z}$ plane discretized into $1160 \mathrm{RWG}$ basis functions each.

Fig. 2 shows the evolution of the estimated and the true relative error as a function of the ACA iterations, for a particular random choice of the initial column. Note the steep peak at step 41 which might lead to a highly premature declaration of convergence. 


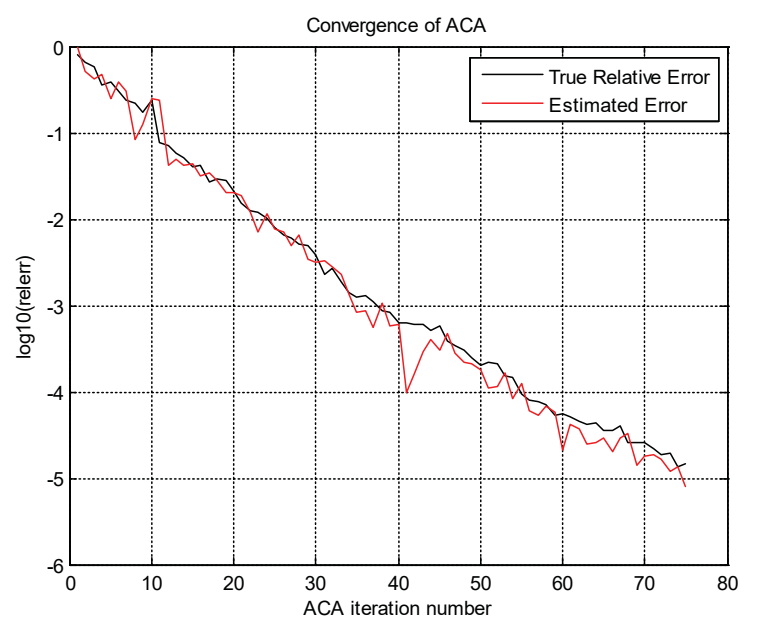

Fig. 2. Example of convergence of ACA relative error for the interaction between the two square plates in Fig. 1.

Fig. 3 shows the distribution of the true relative error after convergence of the ACA algorithm with a threshold of $10^{-4}$, when all 1160 possible random choices of the initial column are tried. Note the variation in the true error of more than one order of magnitude.

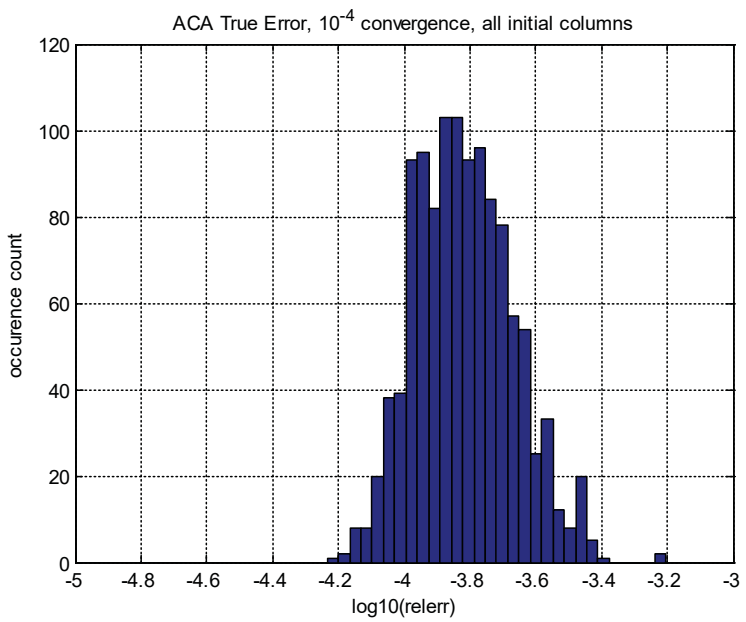

Fig. 3. Distribution of the true relative error after convergence of the ACA algorithm for all 1160 choices of the initial column.

Fig. 4 shows the distribution of the number of iterations corresponding to the 1160 initial choices. Again, the variation is very high.

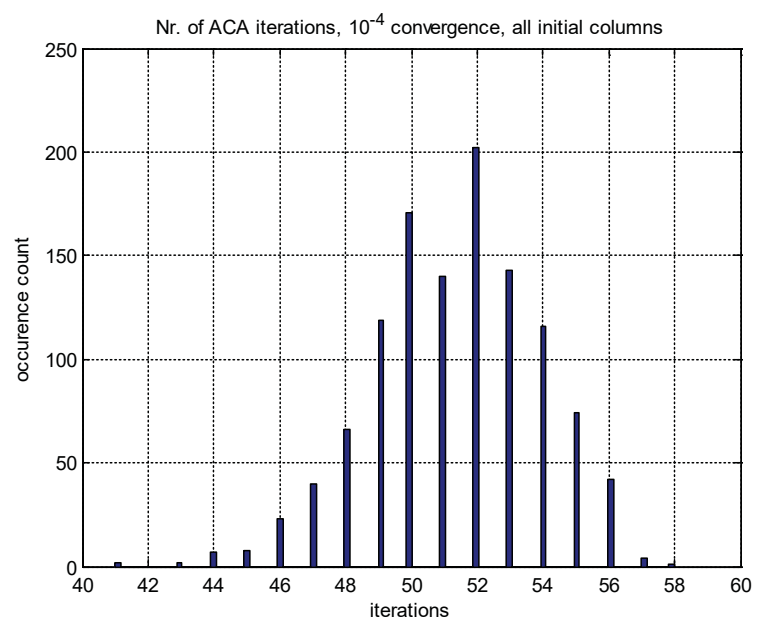

Fig. 4. Distribution of the number of iterations for convergence of the ACA algorithm for all 1160 choices of the initial column.

\section{CONCLUSION}

The aim of this contribution was to draw attention to the often overlooked statistical uncertainty in the ACA algorithm. Presently no consistent solution to this problem has been found. Some observations that may lead the way to such a solution in the future will be presented in the oral presentation at the conference.

\section{REFERENCES}

[1] M. Bebendorf, "Approximation of boundary element matrices," Numerische Matematik, Vol. 86, No. 4, pp. 565-589, 2000.

[2] J. Laviada, R. Mittra, M. R. Pino, and F. Las-Heras, "On the convergence of the ACA," Microwave Opt. Technol. Lett, vol. 51, no. 10, pp. 2458-2460, Oct. 2009

[3] A. Heldring, E. Ubeda and J.M. Rius, 'On the Convergence of the ACA algorithm for Radiation and Scattering Problems,' IEEE Trans. Ant. and Prop, Vol. 62, No. 7, July 2014 